\title{
The G Protein-Coupled Receptor GPR17: Overview and Update
}

\author{
Gabriella Marucci, Diego Dal Ben, Catia Lambertucci, Claudia Santinelli, Andrea Spinaci, \\ Ajiroghene Thomas, Rosaria Volpini, and Michela Buccioni* ${ }^{[a]}$
}

The GPR17 receptor is a G protein-coupled receptor (GPCR) that seems to respond to two unrelated families of endogenous ligands: nucleotide sugars (UDP, UDP-galactose, and UDP-glucose) and cysteinyl leukotrienes ( $\mathrm{LTD}_{4}, \mathrm{LTC}_{4}$, and $\left.\mathrm{LTE}_{4}\right)$, with significant affinity at micromolar and nanomolar concentrations, respectively. This receptor has a broad distribution at the level of the central nervous system (CNS) and is found in neurons and in a subset of oligodendrocyte precursor cells (OPCs). Unfortunately, disparate results emerging from different laboratories have resulted in a lack of clarity with regard to the role of GPR17-targeting ligands in OPC differentiation and in myelination. GPR17 is also highly expressed in organs typically undergoing ischemic damage and has various roles in specific phases of adaptations that follow a stroke. Under such conditions, GPR17 plays a crucial role; in fact, its inhibition decreases the progression of ischemic damage. This review summarizes some important features of this receptor that could be a novel therapeutic target for the treatment of demyelinating diseases and for repairing traumatic injury.

\section{Introduction}

The progress of human genome sequencing has revealed the existence of several hundred orphan $G$ protein-coupled receptors $(\mathrm{GPCRs})^{[1]}$ that are molecularly known receptors but still lack a defined physiologically significant ligand. The deorphanization of these receptors and the identification of their roles could be useful to explain novel regulatory mechanisms of physiological phenomena and to reveal novel drug targets. ${ }^{[1-3]}$

One such receptor, GPR17, also called the P2Y-like receptor and R12, was first characterized by Raport and collaborators ${ }^{[4]}$ in efforts to identify new chemokine receptors. GPR17 has features typical of the GPCR superfamily, such as seven transmembrane domains (TM1-TM7), eight amphipathic helices, and a portion of the sites for $\mathrm{N}$-glycosylation, which is essential for transport from the rough endoplasmic reticulum to the plasma membrane (Figure 1).

Moreover, the intracellular C-terminus has a PDZ-1-like region $(X-S-X-\varnothing)$ that is involved in the interaction of the receptor with signaling proteins during internalization and recycling. Human GPR17 (hGPR17) is about $90 \%$ homologous with the murine (mGPR17) and rat (rGPR17) forms. It was shown, in fact, that in the alignment of the amino acid sequences of rat, mouse, and human GPR17, transmembrane domains TM3, TM6, and TM7 almost completely overlap. The conservation of a typical region, TM6 $(\mathrm{H}-\mathrm{XX}-\mathrm{R})$, which is considered essential

[a] Prof. G. Marucci, Prof. D. Dal Ben, Prof. C. Lambertucci, Dr. C. Santinelli, Dr. A. Spinaci, Dr. A. Thomas, Prof. R. Volpini, Dr. M. Buccioni School of Pharmacy, Medicinal Chemistry Unit, University of Camerino, Via S. Agostino, 1, 62032 Camerino, MC (Italy)

E-mail:michela.buccioni@unicam.it

(iD) The ORCID identification number(s) for the author(s) of this article can be found under http://dx.doi.org/10.1002/cmdc.201600453.

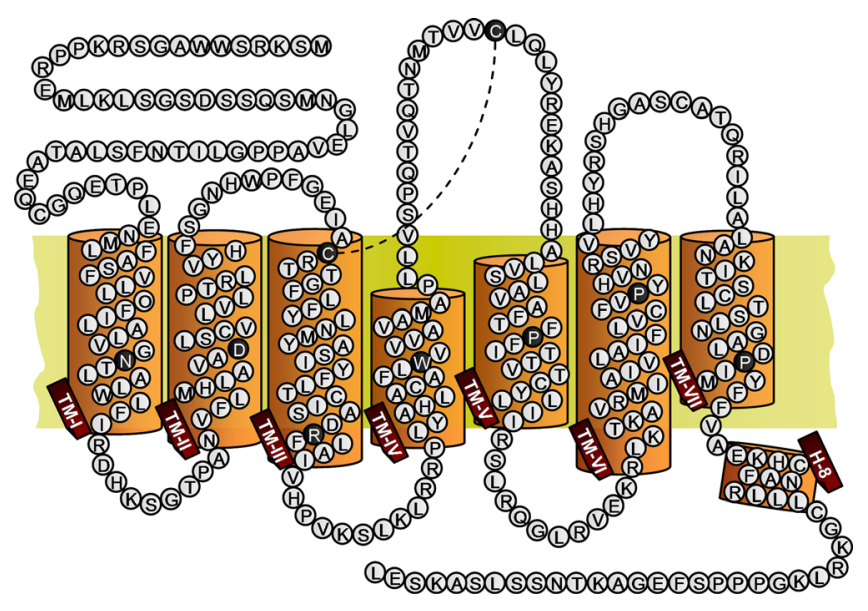

Figure 1. Representation of hGPR17 structure.

for linking with the ligand and is usually present in GPCRs, confirms that GPR17 belongs to the GPCR family. ${ }^{[5-7]}$ From a phylogenetic point of view, the receptor is located in an intermediate position between $\mathrm{P} 2 \mathrm{Y}$ purinergic and cysteinyl leukotriene receptors (CysLTRs), specifically between P2 $\mathrm{Y}_{12 / 13 / 14} \mathrm{Rs}$ and CysLT1/2Rs (Figure 2).

Conflicting literature data (discussed below) make the function of GPR17 less certain, though a preponderance of evidence suggests a dual pharmacological profile. Well-known P2YR and CysLTR antagonists, specifically, the P2Y,R-selective antagonist 2'-deoxy- $N^{6}$-methyladenosine $\quad 3^{\prime}, 5^{\prime}$-biphosphate (MRS 2179), the $P 2 Y_{12 / 13} R$ antagonist $N^{6}$-(2-methyl-thioethyl)-2(3,3,3-trifluoropropylthio)- $\beta, \gamma$-dichloromethylene-ATP (cangrelor), and CysLT1R antagonists montelukast and pranlukast, 


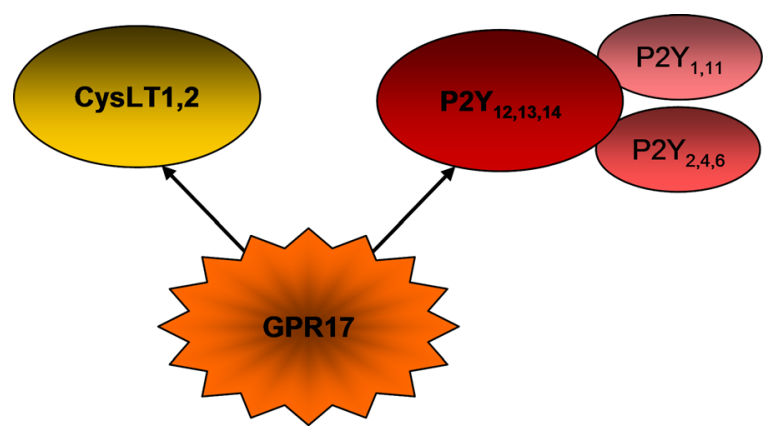

Figure 2. Phylogenetic scheme showing the relationship between GPR17, P2YR, and CysLTR. were found to counteract GPR17 activation in a $\left.{ }^{35} \mathrm{~S}\right] \mathrm{GTP} \gamma \mathrm{S}$ binding assay. ${ }^{[8-11]}$

Cangrelor, which does not act as a GPR17 agonist ${ }^{[8]}$ can instead act as an antagonist, as it completely blocks the activation of GPR17 by UDP-glucose or $\mathrm{LTD}_{4}$ in a concentration-dependent manner. ${ }^{[8,9,11-15]}$ GPR17 ligands, structure, pharmacological activity, and affinity are reported in Table 1.

A homology model of human GPR17 was built using the Xray crystal structure of bovine rhodopsin (bRh) as a template. ${ }^{[16]}$ Two isoforms of the receptor are known, a short form (GPR17S, 339 amino acids) and a long form (GPR17-L, 367 amino acids), which differ in the $\mathrm{N}$-terminus region (Figure 1). ${ }^{[11,16,17]}$

Table 1. Ligand specificity at human (h), rat (r), and mouse (m) GPR17 in $\left.{ }^{35} \mathrm{~S}\right] \mathrm{GTP} \gamma \mathrm{S}$ binding assays performed in 1321N1-transfected cells.

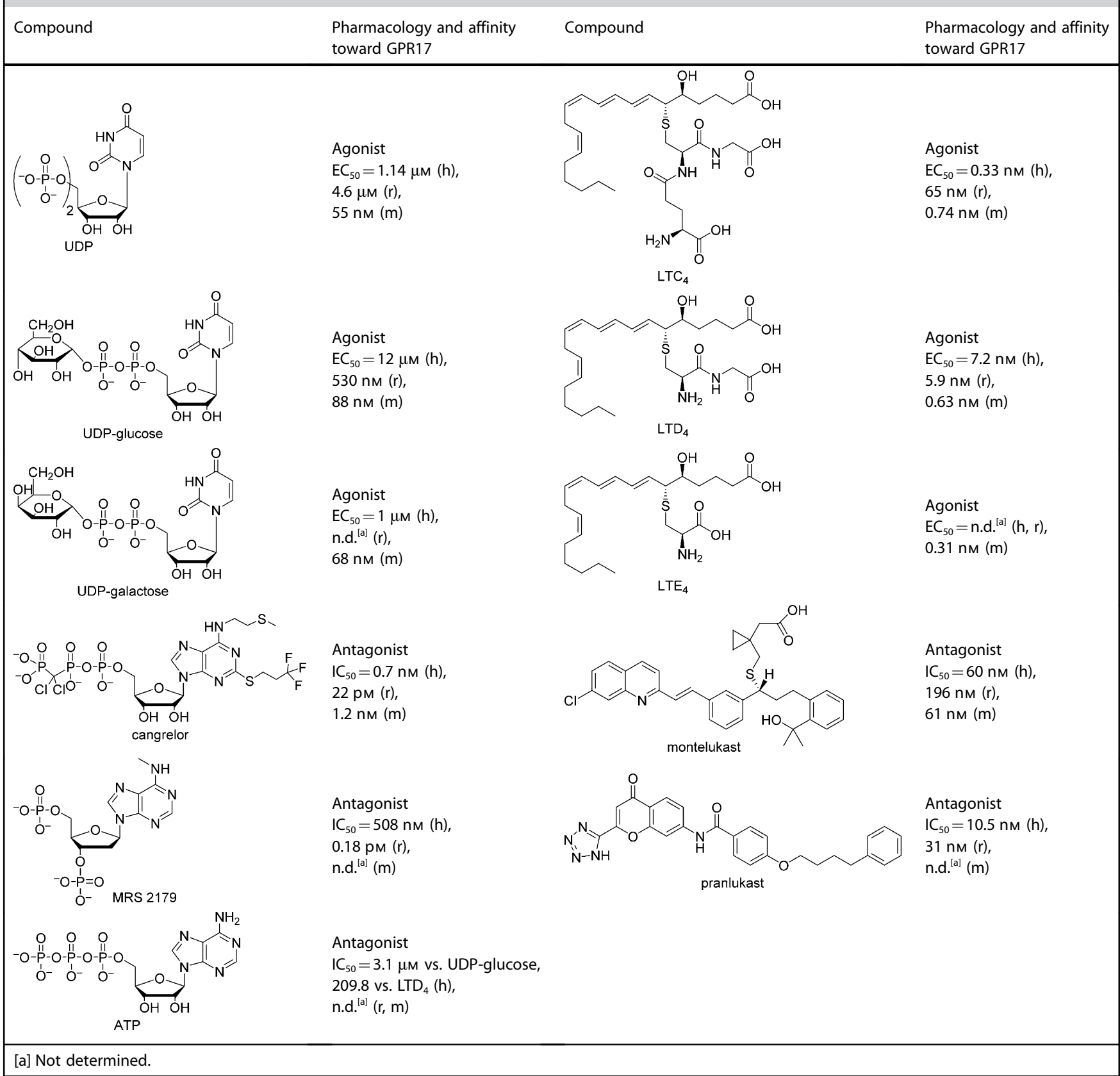


The different functional properties of GPR17 isoforms were partly confirmed by Benned-Jensen and Rosenkilde, who reported that GPR17-L was only minimally activated by uracil nucleotides, except for UDP, which was inactive. Similarly, GPR17$S$ was activated with similar potencies but slightly lower efficacies in comparison with the nucleotide receptors $\mathrm{P}_{2} \mathrm{Y}_{6} \mathrm{R}$ and $\mathrm{P} 2 \mathrm{Y}_{14} \mathrm{R}^{[12]}$

\section{GPR17 localization}

Both rat and human GPR17 are mainly expressed in the CNS, in particular on oligodendrocyte precursor cells (OPCS), and in the kidney and heart, which are organs that typically undergo ischemic damage, while in contrast, there is a minimal expression in the liver and lung. ${ }^{[8,9,18,19,20]}$ Immunohistochemistry studies in mouse brain performed by Lecca and collaborators using a specific homemade anti-GPR17 antibody showed that the GPR17 is expressed by neurons, as demonstrated by costaining with neuronal specific markers (i.e., NeuN and SMI311). ${ }^{[9]}$ On the other hand, Chen and co-workers did not find expression of GPR17 in neurons of adult mouse brain under basal physiological conditions. ${ }^{[18]}$ This discrepancy could be due to the specificity of the GPR17 antibodies used for immunohistochemistry. Moreover, no co-localization was found with cortical astrocytes, as shown by labeling with the specific marker glial fibrillary acidic protein (GFAP). ${ }^{[9,21]}$

In addition, GPR17 is greatly expressed in ependymal cells, which are located in the hippocampus and in direct contact with ventricle cavities. ${ }^{[9]}$ Moreover, no co-localization of more mature myelinating oligodendroglial markers was found with GPR17. This suggests that GPR17 expression is exclusively segregated to the early stages of the oligodendrocyte differentiating pathway, and that the receptor is switched off when these cells achieve functional maturation. Maisel and collaborators demonstrated that gpr17 is one of the most important genes present in human adult neuronal precursor cells (NPCs) ${ }_{i}^{[22]}$ for this purpose, the presence of GPR17 was studied in mouse brain neurogenic areas, i.e., the dentate gyrus (DG) of hippocampus and the subventricular zone (SVZ) of lateral ventricles $(\mathrm{LV}){ }^{\left[{ }^{9]}\right.}$ In the walls of LV, GPR17 is highly expressed in ependymal cells, which are in direct contact with ventricle cavities. The reactive GPR17 cells were also found in the hippocampus, a neurogenic area present in the adult brain. ${ }^{[9]}$ Moreover, it was demonstrated that GPR17 plays a crucial role in oligodendrocyte differentiation. In fact, GPR17 is expressed in primary cortical neuron-glia cultures by OPCs, together with typical pre-oligodendroglial markers like NG2 chondroitin sulfate proteoglycan. $^{[8-10,23,24]}$

The results showed that OPCs expressing GPR17 represent a premyelinating phase of oligodendrocyte differentiation and take part in the brain reaction to acute and chronic lesions. ${ }^{[23,24]}$ Additionally, GPR17 is expressed in the spinal cord, supporting a role for this receptor in both neurons and oligodendrocytes. GPR17 is expressed, under physiological conditions, in neurons and oligodendrocytes at different phases of maturation and in ependymal cells lining the central canal, but it is not expressed in astrocytes. ${ }^{[18,25]}$
It is important to note that the distribution of the two GPR17 splicing variants (short, S, and long, L) are about the same in many different regions of the brain but are less expressed in the thalamus. Expression levels increase in the following order: hypothalamus $<$ cerebellum $<$ amygdala $<$ cerebellar hemisphere $<$ frontal cortex $<$ hippocampus $<$ putamen. Moreover, both isoforms exhibited higher expression in the putamen region with respect to the whole brain. An exception is in the hippocampus area, where hGPR17-S is more highly expressed.

In the peripheral nervous system, the situation is different, as the long isoform (hGPR17-L) is expressed at higher levels in the heart and kidneys, particularly when compared with the brain, where the level of hGPR17-S is very low. This situation indicates that GPR17-S plays a principal role in the brain, while GPR17-L probably adopts this role in the heart and kidneys. This differential expression profile suggests tissue-specific roles in humans. ${ }^{[12]}$

\section{GPR17 pathological role in oligodendrocyte maturation and myelination processes}

As GPR17 is expressed in organs typically undergoing ischemic damage, its role was explored in a rat animal model of ischemic stroke (rat middle cerebral artery occlusion (MCAo) $)^{[8]}$ and in a mouse model. ${ }^{[9]}$ GPR17 plays a differential role that seems to depend on specific phases of response after stroke (sequentially, death of irreversibly damaged cells, clearance of dead cells, remodeling, and repair). Upon ischemia induction, there is a time-dependent upregulation of GPR17, with rapid expression in neuronal cells nearest the injured area.

Twenty-four hours after MCAo, various neurons overexpress GPR17; at the same time, co-presence of HSP70, an inducible marker of cellular stress, injury, and death, suggests that the GPR17 upregulation is correlated with neuronal death. This finding was confirmed $48 \mathrm{~h}$ after injury, as the lesion site no longer presented neurons expressing GPR17, presuming the death of the neurons overexpressing this receptor. The situation is different $48-72 \mathrm{~h}$ after MCAo; in fact, many cells expressing GPR17 and a marker of activated microglia/macrophage (IB4) were present at the borders of the lesioned area. $^{[26-28]}$

Seventy-two hours after MCAo, a greater number of OPCs expressing GPR17 was found in the regions surrounding the ischemic area and in the ipsilateral corpus striatum, ${ }^{[9]}$ suggesting that the proliferation or recruitment of OPC increased in response to demyelination. Administration of cangrelor or montelukast, two GPR17 antagonists, during acute injury caused a reduction in infarct ischemic area. ${ }^{[8,9]}$ The administration of GPR17 UDP-glucose agonist in a neonatal rat model of ischemic periventricular leukomalacia (PVL), in contrast, resulted in the recovery of myelin sheaths and improved motor functions. ${ }^{[29-31]}$ This incongruity could be due to the different consequence of the ischemic insult in neonatal brain compared with adults.

In line with these findings, some authors have demonstrated that oligodendrocytes are very susceptible to brain damage 
and that proliferation of OPCs is identified after ischemia nearby the lesioned area, ${ }^{[32]}$ likely as a response to demyelination. ${ }^{[33,34]}$ It is important to note that in the first two weeks after ischemia, OPCs collect in the peri-infarct area and then differentiate into mature oligodendrocytes. ${ }^{[35,36]}$ These findings suggest that GPR17 could be considered a biosensor that is stimulated by brain injury in several embryonically distinct cell types with two different time-dependent implications: firstly, contributing to evolution of the damage, and secondly, in the remodeling and repair of damage. These assumptions are supported, moreover, by the role played by endogenous cysteinyl leukotrienes (CysLTs) and nucleotides, natural ligands of GPR17, in the injured brain. In fact, following the release of these molecules from damaged cells, they stimulate the brain through binding with specific receptors with the objective of resisting brain damage and, at the same time, initiating repair mechanisms.

Additional studies were performed on spinal cord injury $(\mathrm{SCl})$ in order to analyze the different time-expression changes of GPR17. ${ }^{[13]}$ Results showed that SCl induced an intense and rapid death of neurons and oligodendrocytes that express GPR17 inside the lesioned area. Successively, $24 \mathrm{~h}$ after SCl, activated microglia/macrophages migrated toward and infiltrated the lesioned area, and their number increased over time after injury.

One week after $\mathrm{SCl}$, this receptor expression decreases, suggesting that the expression of GPR17 is augmented during the early phases of microglia/macrophage activation, probably due to enhanced migration to and infiltration of the lesioned area, followed by downregulation at the end of these processes. GPR17 is expressed on ependymal cells lining the central canal, which could support the apparent opposite effects (i.e., cell death and damage repair) mediated by the receptor. ${ }^{[37]}$

The ependymal cells can be considered the stem cells of the adult spinal cord, because after injury, they start to proliferate and to generate astrocytes and oligodendrocytes that migrate to the lesion area constructing a significant part of the glial cicatrix. ${ }^{[38]}$ In the brain, the usually quiescent ependymal cells can also generate neuroblasts and astrocytes after stroke. ${ }^{[39]}$ As GPR17 is expressed in ependymal cells, it could play a role in driving precursor cell specification and differentiation after brain injury and $\mathrm{SCl}$.

GPR17 seems to be implicated in demyelinating diseases and represents one of the first potential GPCR drug targets for demyelinating pathologies of the CNS, such as multiple sclerosis (MS). In the case of loss of oligodendrocytes and myelin, axonal functions are impaired; therefore, in pathologies like MS, cerebral ischemia, or stroke, new oligodendrocytes are recruited to replace the injured cells and form new myelin sheets. ${ }^{[40]}$

After a demyelinating event, there is a spontaneous brain repair process that can lead to a limited positive neurological outcome. ${ }^{[41]}$ Therefore, it is crucial to promote oligodendrogenesis to facilitate the natural brain repair process. Preclinical studies confirm that enhancement of endogenous oligodendrogenesis in these pathologies by pharmacological and cellbased therapies facilitates brain repair processes and reduces neurological deficits. It is broadly known and shared by the entire scientific community involved in the study of GPR17 that this receptor is implicated in the remyelination process, but the issue regarding whether such desired pharmacological effects are due to its activation or inhibition remains unresolved. Studies performed in GPR17-overexpressing mice showed a strong demyelination process, while in the knockout variant, the myelination process increased. ${ }^{[18]}$ Furthermore, researchers demonstrated that GPR17 is mainly expressed by OPCs at different levels, depending on their differentiation state, but in the spinal cord and optic nerve, GPR17 is downregulated during the peak period of myelination and in adulthood. ${ }^{[18,19,42]}$

Moreover, it was demonstrated that in a mouse model of MS (demyelination injury induced by experimental autoimmune encephalomyelitis [EAE]) that GPR17 is upregulated in the CNS region where demyelination was occurring. ${ }^{[43]}$ As GPR17 expression is downregulated during active myelinogenesis and upregulated in demyelinating lesions, chances are that the remarkable expression of this receptor could negatively affect oligodendrocyte myelination. Moreover, experiments performed to treat cerebellar slice cultures of mice from their postnatal day with a selective agonist of GPR17 produced a block of oligodendrocyte maturation and myelination arrest, due to loss of myelin basic protein (MBP)-positive cells, ${ }^{[44,45]}$ underlining a negative involvement of GPR17 in CNS repair mechanisms. It was also demonstrated that the CysLTR antagonists montelukast and pranlukast have a neuroprotective effect on cerebral ischemia in rats and mice, proving their usefulness in the treatment of cerebral ischemia at earlier phases. ${ }^{[46]}$

Moreover, the administration of montelukast produced an increase in the proliferation rate of stem cells, leading to a major pool of cells in the undifferentiated stage. ${ }^{[47]}$ In line with these findings, administration of UDP-glucose, UDP, or $\mathrm{LTD}_{4}$ in neurospheres from murine oligodendroglioma cells produced a decrease in the number of Olig2 $p$ oligodendrocytes, emphasizing that GPR17 activation promotes cell differentiation. ${ }^{[45]}$

Recently, Simon and collaborators demonstrated that when GPR17 is stimulated by an agonist, specifically MDL29951 (Figure 3), there is a decrease in MBP expression levels, mainly by the $G_{\alpha i / o}$ signaling pathway, and a subsequent diminution of adenylyl cyclase-cAMP-PKA-cAMP response element-binding protein (CREB) ${ }^{[48]}$

In contrast, findings obtained

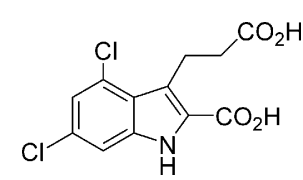

from other researchers through genetic and pharmacological studies emphasize that GPR17 activation (by UDP-glucose or LTD 4 ) promotes the transition of OPCs from a pre-immature phenotype to fully mature cells expressing MBP. Furthermore, inhibition of the receptor using P2YR or CysLTR antagonists (cangrelor or montelukast) or specific silencing RNAs inhibited OPC maturation. In some knockout experiments and in cultured OPCs, it was demonstrated that GPR17 promoted cell differentiation, al- 
though when the cells expressed the pre-oligodendrocyte marker O4, GPR17 had to be downregulated to allow complete differentiation. This confirms that GPR17 is not present on MBP-expressing mature oligodendrocytes..$^{[8,9,17,23]}$ Additionally, it was proven that the activation of $\mathrm{K}^{+}$currents, both in a subpopulation of OPCs and in immature $\mathrm{O}^{+}$pre-oligodendrocytes, is mediated by GPR17. In contrast, this event is not present in mature oligodendrocytes. This correlates with the GPR17 pattern of dissimilar expression during the maturation of OPCs; in fact, GPR17 was shown to gradually disappear in late OPCs. ${ }^{[49]}$

There is evidence of a probable relationship between GPR17 and $\mathrm{P} 2 \mathrm{Y} 12$ that plays an important role in cell myelination processes. In fact, when GPR17 is downregulated, P2Y12 is upregulated, and the activation of GPR17 promotes cell differentiation toward myelination, while the activation of P2Y12 leads to cell maturation. ${ }^{[50]}$ More recently, by means of the first GPR17 transgenic reporter mouse, Viganò and collaborators verified that OPCs express GPR17 in their earlier life. The confocal microscopy technique used made it possible to visualize the final destiny of GPR17-expressing precursors. ${ }^{[51]}$ The presence of the receptor during the maturation of OPCs seems to have a reduction in a precise maturation time; in fact, its forced expression at the level of pre-oligodendrocytes led to a decrease in the maturation and myelination processes, while gpr 17 gene silencing promoted the maturation of OPCs to myelinating cells. ${ }^{[9,19,27]}$

The ability of GPR17 to organize lesion remodeling and repair in human brain was also demonstrated. ${ }^{[38]}$ The receptor characterization in human brain was performed in neurosurgical and autoptic samples from patients with traumatic brain injury (TBI). This resulted in overexpression of the receptor in OPCs as a consequence of a local injury, probably due to the release of diffusible factors from damaged cells, as demonstrated by the fact that overexpression in OPCs was found in all patients who had died of intracranial complications and only in $20 \%$ of patients who died of extracranial complications. In addition, there was a negative correlation between GPR17 expression and post-traumatic survival time; in fact, patients who died after more than 16 days following injury showed a clear decrease in receptor expression.

In addition to the role of GPR17 in oligodendrogenesis, a possible implication in allergic pulmonary inflammation has been reported. Maekawa and collaborators demonstrated that GPR17 negatively regulates the levels of IgE and specific $\lg G 1$ in serum, and the inflammatory cell accumulation in the lung and in the bronchoalveolar lavage fluid after CysLT1R activation. The same report further showed a negative modulation of Th2/Th17 cytokine expression by GPR17 in the lung after intranasal sensitization with a house dust mite (extract of Dermatophagoides farinae [Df]) in mice.

Sensitization of wild-type recipients with Df pulsed in dendritic cells derived from bone marrow of each genotype revealed an appreciable increase in inflammation of the lungs and serum IgE in GPR17-deficient mice in comparison with the wild-type strains; in contrast, there were decreased responses in CysLT1R-deficient mice. These results highlight a constitutive downregulation of CysLT1R functions due to GPR17 activation in both antigen presentation and downstream stages of allergic pulmonary inflammation. ${ }^{[52]}$ Based on these results, GPR17 appears to play a key role in allergic pulmonary inflammation and also clearly represents a novel target for new TBI therapies, even if its role in the oligodendrogenesis process still remains to be elucidated.

\section{Constitutive and ligand-mediated activities of GPR17}

As reported above, GPR17 seems to be activated by CysLTs and nucleotide sugars, with the inhibition of CAMP production and intracellular $\mathrm{Ca}^{2+}$ accumulation. Unfortunately, there are discrepancies between different laboratories, with some reporting that GPR17 responds to CysLTs and uracil nucleotides, ${ }^{[8,53]}$ and others reporting that it is only activated by CysLTs ${ }^{[44]}$ or uracil nucleotide ligands. ${ }^{[54]}$ Furthermore, a number of researchers also assert that GPR17 does not respond to either of the two families of ligands. ${ }^{[55]}$

According to Ciana and co-workers, $\mathrm{LTC}_{4}$ and $\mathrm{LTD}_{4}$ both activate $\mathrm{GPR} 17$ with $\mathrm{EC}_{50}$ values in the nanomolar range ${ }^{[8,56]}$ while UDP, UDP-glucose, and UDP-galactose activate GPR17 with $\mathrm{EC}_{50}$ values in the micromolar range. Experiments were carried out in several cell lines using both $\left[{ }^{35} \mathrm{~S}\right] \mathrm{GTP} \gamma \mathrm{S}$ binding to purified membranes and a CAMP accumulation assay in live cells using a competitive protein binding method, yielding results that demonstrated agreement between the two approaches. $^{[8,57]}$ To demonstrate the receptor coupling to a $G_{i}$ protein, the authors performed the same experiments in cells pretreated with the $G_{i}$ inhibitor pertussis toxin. The toxin fully inhibited the $\left.{ }^{35} \mathrm{~S}\right] \mathrm{GTP} \gamma \mathrm{S}$ binding stimulated by agonists and fully suppressed the agonist effects on forskolin-induced CAMP formation, indicating a crucial role for $G_{i}$ protein in GPR17 responses. The activation of GPR17 by CysLTs has not yet been verified by independent laboratories and thus remain a controversial matter, even though work published by Marschallinger and co-workers suggests that the effects of montelukast, a well-known antagonist of CysLT1R, on neurogenesis and cognition are most likely mediated through inhibition of GPR17, suggesting a possible involvement of CysLTs in receptor activation. ${ }^{[58]}$ Moreover, there is further evidence regarding the interaction between CysLTs and GPR17 reported by Ren et al., ${ }^{[59]}$ with the authors affirming that when $\mathrm{LTD}_{4}$ was injected directly into the third ventricle of mice, it was able to activate GPR17induced food intake. In contrast, administration of cangrelor produced a significant decrease in food intake.

Buccioni and collaborators achieved similar results, although their experiments were performed using different methodologies. In fact, uracil nucleotides and nucleotide sugars promoted inhibition of CAMP production with $\mathrm{EC}_{50}$ values similar to those reported by Ciana and co-workers. ${ }^{[53]}$ Both laboratories tested selective antagonists for both classes of receptor family (CysLTRs and purinergic receptors), and the $\mathrm{IC}_{50}$ values (manuscript in preparation) obtained from an $\left[{ }^{35} \mathrm{~S}\right] \mathrm{GTP} \gamma \mathrm{S}$ binding assay ${ }^{[8]}$ and a GloSensor cAMP assay ${ }^{[53]}$ showed a strong correlation.

The ability of GPR17 to bind purinergic ligands was also demonstrated by Temporini and co-workers through a new 
technique: frontal affinity chromatography-mass spectrometry (FAC-MS). A liquid chromatography stationary phase containing immobilized membranes from $1321 \mathrm{~N} 1$ cells expressing GPR17 was used to reveal the specific interaction between the receptor and three ligands that were reported to interact with GPR17: cangrelor (a P2 $\mathrm{Y}_{12 / 13} \mathrm{R}$ antagonist), MRS2179 (a P2Y $\mathrm{R}$ antagonist), and the agonist UDP (Table 1$){ }^{[8]}$ The validity of the experiment was confirmed by the agreement of the calculated dissociation constant $\left(K_{\mathrm{d}}\right)$ values with previously reported data. ${ }^{[60]}$ This technique was also used to screen a library of nucleotide derivatives in order to select high-affinity ligands and to identify a lead compound for the generation of new potent and selective ligands. The chromatographic results were validated with the $\left.{ }^{35} \mathrm{~S}\right] \mathrm{GTP} \gamma \mathrm{S}$ binding assay. In particular, the library for GPR17 ligands was selected, taking into account the chemical structures of the three above-mentioned known receptor ligands: cangrelor, MRS2179, and UDP. A series of ATP derivatives, substituted at the 2- and/or $N^{6}$-position, were tested (3-7). Furthermore, in order to study the importance of the 3-nitrogen purine ring, the $2, N^{6}$-disubstituted ATP 3-deaza analogue was evaluated (5), in addition to a bisphosphate derivative analogue of MRS2179 (2) and a 5-substituted UDP analogue (1). The results are reported in Table 2. Comparing the results obtained with FACS-MS with those obtained with $\left.{ }^{[5} \mathrm{S}\right] \mathrm{GTP} \gamma \mathrm{S}$, it is noted that the elution order of the analytes by column is closely related to the activity obtained with the binding assay, expressed as half-maximal response concentrations $\left(\mathrm{EC}_{50}\right)$ or half-maximal inhibition $\left(\mathrm{IC}_{50}\right)$ values for agonists and antagonists, respectively. ${ }^{[61]}$

Experimental results from Hennen and collaborators led to conclusions different from those obtained by other research groups. Hennen et al. tested some of the P2YR agonists mentioned above, but all of them were unable to activate GPR17. This group also identified a small molecule, MDL29951 (Figure 3), that was able to activate the receptor with remarkable selectivity, even in an environment of endogenous purinergic receptors. ${ }^{[4,62]}$ This molecule is the unique ligand whose ability to activate GPR17 is undisputed. The effect of MDL29951 was hampered by the CysLT1R antagonist pranlukast. Unlike montelukast, which resulted in total inactivity,

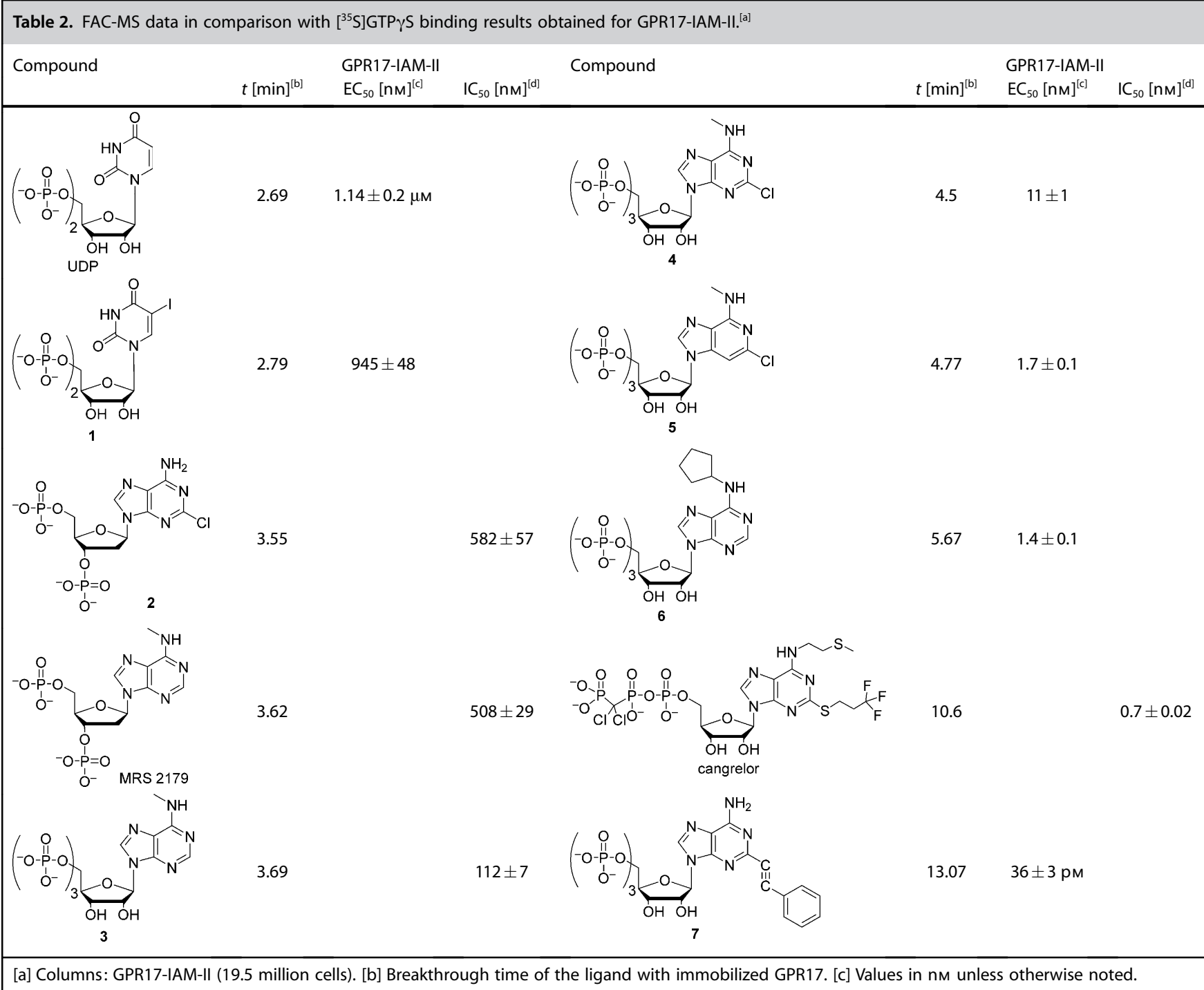


pranlukast was able to inhibit GPR17 in a surmountable and noncompetitive manner.

Data published by Jensen and co-workers were not able to confirm the activation of GPR17 by CysLTR agonists. ${ }^{[12,54]}$ These authors performed $\left.{ }^{35} \mathrm{~S}\right] \mathrm{GTP} \gamma \mathrm{S}$ binding assays in two different cell lines expressing the long or the short isoform of hGPR17, but $\mathrm{LTD}_{4}$ did not bind to any of these isoforms. In addition, Jensen and colleagues tested montelukast, previously reported as an antagonist of both CysLT1R and GPR17, ${ }^{[11]}$ but did not observe any activity. Subsequently, the same compound was tested as an inverse agonist on the two GPR17 isoforms, but it did not suppress the constitutive activity of the receptor.

Different results were obtained when the ability of some P2YR agonists to activate the two isoforms was tested. UDP, UDP-galactose, and UDP-glucose activated hGPR17-S with $\mathrm{EC}_{50}$ values similar to those reported in literature for this isoform, ${ }^{[8]}$ while the two UDP-sugars were much less potent toward hGPR17-L, and UDP was not able to activate this isoform at all. In view of the fact that different research groups affirm that GPR17 activation is antagonized by several P2YR ligands, ${ }^{[8,11,57]}$ the $P 2 Y_{12} R$ antagonist ticlopidine was studied as inverse agonist at GPR17. Ticlopidine was not able to antagonize the effect of UDP-glucose or UDP-galactose, nor was it able to suppress the constitutive activities of the two isoforms.

Unlike previously reported results, Qi and collaborators asserted that neither purinergic nor CysLTRs ligands activate GPR17. These researchers investigated receptor activation by measuring inositol phosphate output, CAMP production inhibition, and $\mathrm{Ca}^{2+}$ mobilization in five different cell lines stably or transiently expressing the receptor. Experiments were also performed to co-express GPR17 and CysLT1R. In agreement with Maekawa's results, Qi and co-workers noted that when the two receptors were co-expressed, a clear reduction in CysLT1R activity was observed, along with a reduction in expression of the same receptor at the cell surface, emphasizing that GPR17 behaves as a negative regulator of CysLT1R. ${ }^{[5]}$ These data are in agreement with a recent study by Maekawa et al., which demonstrated that GPR17 knockout mice demonstrate an increased immune response to dust mites with augmented levels of IgE and inflammatory cytokines, as they lack GPR17induced inhibition of CysLT1R activity, as previously reported. ${ }^{[52,63]}$

\section{Conclusions}

Several questions remain unanswered. There is disagreement regarding GPR17 ligand agonist or antagonist profiles, and the role of this receptor in the pathologies of the nervous system is not completely clarified. The identification of purinergic ligands, which include agonists and antagonists, and the small molecule MDL29951, a GPR17 agonist, increased the possibility of carrying out the investigation of GPR17 pathophysiological role. Moreover, the activation of GPR17 by CysLTs is highly controversial, even though Marschallinger and co-workers suggest that the effects of montelukast on neurogenesis and cognition are most likely mediated through inhibition of GPR17. On the other hand, the high expression of this receptor in organs that usually undergo ischemic damage, its key role in oligodendrogenesis, and some available data on the up- or downregulation of GPR17 during myelination induction make this receptor a novel therapeutic tool in the treatment of demyelinating diseases such as MS. In light of this information, it is very difficult to unequivocally explain the inconsistencies regarding the natural ligands of GPR17. Research efforts should be focused on clarifying the role of this receptor in neuropathology and in discovering new compounds able to interact with GPR17.

Keywords: G protein-coupled receptors - leukotrienes membrane proteins - myelination - oligodendrocyte precursor cells (OPCs)

[1] R. Fredriksson, M. C. Lagerström, L. G. Lundin, H. B. Schiöth, Mol. Pharmacol. 2003, 63, 1256-1272.

[2] A. D. Howard, G. McAllister, S. D. Feighner, Q. Liu, R. P. Nargund, L. H. Van der Ploeg, A. A. Patchett, Trends Pharmacol. Sci. 2001, 22, 132-140.

[3] K. Mori, M. Miyazato, T. Ida, N. Murakami, R. Serino, Y. Ueta, M. Kojima, K. Kangawa, EMBO J. 2005, 24, 325-335.

[4] C. J. Raport, V. L. Schweickart, D. Chantry, R. L. Eddy, Jr., T. B. Shows, R. Godiska, P. W. Gray, J. Leukocyte Biol. 1996, 59, 18-23.

[5] L. Erb, R. Garrad, Y. Wang, T. Quinn, J. T. Turner, G. A. Weiseman, J. Biol. Chem. 1995, 270, 4185-4188.

[6] Q. Jiang, D. Guo, B. X. Lee, A. M. Van Rhee, Y. C. Kim, R. A. Nicholas, J. B. Schachter, T. K. Harden, K. A. Jacobson, Mol. Pharmacol. 1997, 52, 499507.

[7] K. A. Jacobson, M. F. Jarvis, M. Williams, J. Med. Chem. 2002, 45, 40574093.

[8] P. Ciana, M. Fumagalli, M. L. Trincavelli, C. Verderio, P. Rosa, D. Lecca, S. Ferrario, C. Parravicini, V. Capra, P. Gelosa, U. Guerrini, S. Belcredito, M. Cimino, L. Sironi, E. Tremoli, G. E. Rovati, C. Martini, M. P. Abbracchio, EMBO J. 2006, 25, 4615-4627.

[9] D. Lecca, M. L. Trincavelli, P. Gelosa, L. Sironi, P. Ciana, M. Fumagalli, G. Villa, C. Verderio, C. Grumelli, U. Guerrini, E. Tremoli, P. Rosa, S. Cuboni, C. Martini, A. Buffo, M. Cimino, M. P. Abbracchio, PLoS One 2008, 3, e3579.

[10] A. Fratangeli, E. Parmigiani, M. Fumagalli, D. Lecca, R. Benfante, M. Passafaro, A. Buffo, M. P. Abbracchio, P. Rosa, J. Biol. Chem. 2013, 288, $5241-5256$.

[11] A. M. Pugliese, M. L. Trincavelli, D. Lecca, E. Coppi, M. Fumagalli, S. Ferrario, P. Failli, S. Daniele, C. Martini, F. Pedata, M. P. Abbracchio, Am. J. Physiol. Cell Physiol. 2009, 297, C1028-C1040.

[12] T. Benned-Jensen, M. M. Rosenkilde, Br. J. Pharmacol. 2010, 159, 1092 1105.

[13] S. Daniele, M. L. Trincavelli, M. Fumagalli, E. Zappelli, D. Lecca, E. Bonfanti, P. Campiglia, M. P. Abbracchio, C. Martini, Cell. Signalling 2014, 26, $1310-1325$.

[14] L. Mamedova, V. Capra, M. R. Accomazzo, Z. G. Gao, S. Ferrario, M. Fumagalli, M. P. Abbracchio, G. E. Rovati, K. A. Jacobson, Biochem. Pharmacol. 2005, 71, 115-125.

[15] S. Paruchuri, H. Tashimo, C. Feng, A. Maekawa, W. Xing, Y. Jiang, Y. Kanaoka, P. Conley, J. A. Boyce, J. Exp. Med. 2009, 206, 2543-2555.

[16] C. Parravicini, M. P. Abbracchio, P. Fantucci, G. Ranghino, BMC Struct. Biol. 2010, 10, 8.

[17] I. Eberini, S. Daniele, C. Parravicini, C. Sensi, M. L. Trincavelli, C. Martini, M. P. Abbracchio, J. Comput.-Aided Mol. Des. 2011, 25, 743-752.

[18] Y. Chen, H. Wu, S. Wang, H. Koito, J. Li, F. Ye, J. Hoang, S. S. Escobar, A. Gow, H. A. Arnett, B. D. Trapp, N. J. Karandikar, J. Hsieh, Q. R. Lu, Nat. Neurosci. 2009, 12, 1398- 1406.

[19] M. Fumagalli, S. Daniele, D. Lecca, P. R. Lee, C. Parravicini, R. D. Fields, P. Rosa, F. Antonucci, C. Verderio, M. L. Trincavelli, P. Bramanti, C. Martini, M. P. Abbracchio, J. Biol. Chem. 2011, 286, 10593-10604.

[20] S. Cosentino, L. Castiglioni, F. Colazzo, E. Nobili, E. Tremoli, P. Rosa, M. P. Abbracchio, L. Sironi, M. Pesce, J. Cell. Mol. Med. 2014, 18, 1785-1796.

[21] S. Ceruti, F. Viganò, E. Boda, S. Ferrario, G. Magni, M. Boccazzi, P. Rosa, A. Buffo, M. P. Abbracchio, Glia 2011, 59, 363-378. 
[22] M. Maisel, A. Herr, J. Milosevic, A. Hermann, H. J. Habish, S. Schwarz, M. Kirsch, G. Antoniadis, R. Brenner, S. Hallmeyer-Elgner, H. Lerche, J. Schwarz, A. Storch, Stem Cells 2007, 25, 1231-1240.

[23] M. Fumagalli, D. Lecca, M. P. Abbracchio, Front. Biosci. 2011, 16, 2326 2341.

[24] E. Coppi, L. Cellai, G. Maraula, A. M. Pugliese, F. Pedata, Neuropharmacology 2013, 73, 301-310.

[25] W. A. Tyler, M. R. Jain, S. E. Cifelli, Q. Li, L. Ku, Y. Feng, H. Li, T. L. Wood, Glia 2011, 59, 1754-1769.

[26] E. Boda, F. Vigano, P. Rosa, M. Fumagalli, V. Labat-Gest, F. Tempia, M. P. Abbracchio, L. Dimou, A. Buffo, Glia 2011, 59, 1958-1973.

[27] S. Ceruti, G. Villa, T. Genovese, E. Mazzon, R. Longhi, P. Rosa, P. Bramanti, S. Cuzzocrea, M. P. Abbracchio, Brain 2009, 132, 2206-2218.

[28] B. Zhao, C. Z. Zhao, X. Y. Zhang, X. Q. Huang, W. Z. Shi, S. H. Fang, Y. B. Lu, W. P. Zhang, Q. Xia, E. Q. Wei, Neuroscience 2012, 202, 42-57.

[29] F. X. Mao, W. J. Li, H. J. Chen, L. H. Qian, J. S. Buzby, Brain Res. 2012 1486, $112-120$.

[30] W. J. Li, F. X. Mao, H. J. Chen, L. H. Qian, J. S. Buzby, Neuroscience 2015 $284,444-458$

[31] F. X. Mao, W. J. Li, H. J. Chen, L. H. Qian, J. S. Buzby, Brain Res. 2013 , 1535, 38-51.

[32] R. Gregersen, T. Christiensen, E. Lehrmann, N. H. Diemer, B. Finsen, Exp. Brain Res. 2001, 138, 384-392.

[33] N. G. Bauer, C. Richter-Landsberg, C. French-Constant, Glia 2009, 57, $1691-1705$

[34] F. Biname, D. Sakry, L. Dimou, V. Jolivel, J. Trotter, J. Neurosci. 2013, 33, $10858-10874$

[35] Z. G. Zhang, J. Cereb. Blood Flow Metab. 2011, 31, 614-625.

[36] R. L. Zhang, M. Chopp, C. Roberts, M. Wei, X. Wang, X. Liu, M. Lu, Z. G. Zhang, PLoS One 2012, 7, e48141.

[37] H. Franke, C. Parravicini, D. Lecca, E. R. Zanier, C. Heine, K. Bremicker, M. Fumagalli, P. Rosa, L. Longhi, N. Stocchetti, M. G. De Simoni, M. Weber, M. P. Abbracchio, Purinergic Signalling 2013, 9, 451-462.

[38] K. Meletis, F. Barnabé-Heider, M. Carlén, E. Evergren, N. Tomilin, O. Shupliakov, J. Frisén, PLoS Biol. 2008, 6, e182.

[39] M. Carlén, K. Meletis, C. Göritz, V. Darsalia, E. Evergren, K. Tanigaki, M. Amendola, F. Barnabé-Heider, M. S. Yeung, L. Naldini, T. Honjo, Z. Kokaia O. Shupliakov, R. M. Cassidy, O. Lindvall, J. Frisén, Nat. Neurosci. 2009 12, 259-267.

[40] R. Zhang, M. Chopp, Z. G. Zhang, Front. Cell. Neurosci. 2013, 7, 201.

[41] L. I. Benowitz, S. T. Carmichael, Neurobiol. Dis. 2010, 37, 259-266.

[42] J. McQueen, M. M. Reimer, P. R. Holland, Y. Manso, M. McLaughlin, J. H. Fowler, K. Horsburgh, PLoS One 2014, 9, e87227.

[43] M. Fumagalli, E. Bonfanti, S. Daniele, E. Zappelli, D. Lecca, C. Martini, M. L. Trincavelli, M. P. Abbracchio, Glia 2015, 63, 2327-2339.

[44] S. Hennen, H. Wang, L. Peters, N. Merten, K. Simon, A. Spinrath, S. Blättermann, R. Akkari, R. Schrage, R. Schröder, D. Schulz, C. Vermeiren, K. Zimmermann, S. Kehraus, C. Drewke, A. Pfeifer, G. M. König, K. Mohr, M. Gillard, C. E. Müller, Q. R. Lu, J. Gomeza, E. Kostenis, Sci. Signaling 2013 6, ra93.

[45] J. D. Dougherty, E. I. Fomchenko, A. A. Akuffo, E. Schmidt, K. Y. Helmy, E. Bazzoli, C. W. Brennan, E. C. Holland, A. Milosevic, Cancer Res. 2012, 72, $4856-4868$
[46] G. L. Yu， E. Q. Wei， S. H. Zhang, H. M. Xu， L. S. Chu，W.P. Zhang，Q. Zhang, Z. Chen, R. H. Mei, M. H. Zhao, Pharmacology 2005, 73, 31-40.

[47] C. Huber, J. Marschallinger, H. Tempfer, T. Furtner, S. Couillard-Despres, H. C. Bauer, F. J. Rivera, L. Aigner, Cell. Physiol. Biochem. 2011, 28, $793-$ 804.

[48] K. Simon, S. Hennen, N. Marten, S. Blättermann, M. Gillard, E. Kostenis, J. Gomeza, J. Biol. Chem. 2016, 291, 705-718.

[49] E. Coppi, G. Maraula, M. Fumagalli, P. Failli, L. Cellai, E. Bonfanti, L. Mazzoni, R. Coppini, M. P. Abbracchio, F. Pedata, A. M. Pugliese, Glia 2013 $61,1155-1171$.

[50] M. Fumagalli, D. Lecca, M. P. Abbracchio, Neuropharmacology 2016, 104, $82-93$

[51] F. Viganò, S. Schneider, M. Cimino, E. Bonfanti, P. Gelosa, L. Sironi, M. P. Abbracchio, L. Dimou, Glia 2016, 64, 287-299.

[52] A. Maekawa, W. Xing, K. F. Austen, Y. Kanaoka, J. Immunol. 2010, 185, $1846-1854$

[53] M. Buccioni, G. Marucci, D. Dal Ben, D. Giacobbe, C. Lambertucci, L. Soverchia, A. Thomas, R. Volpini, G. Cristalli, Purinergic Signalling 2011, 7 , $463-468$.

[54] C. Norregaard, T. Benned-Jensen, M. M. Rosenkilde, Curr. Top. Med. Chem. 2011, 11, 618-628.

[55] A. D. Qi, T. K. Harden, R. A. Nicholas, J. Pharmacol. Exp. Ther. 2013, 347, $38-46$.

[56] M. Bäck, W. S. Powell, S. E. Dahlén, J. M. Drazen, J. F. Evans, C. N. Serhan, T. Shimizu, T. Yokomizo, G. E. Rovati, Br. J. Pharmacol. 2014, 171, 3551 3574

[57] S. Daniele, M. L. Trincavelli, P. Gabelloni, D. Lecca, P. Rosa, M. P. Abbracchio, C. Martini, J. Pharmacol. Exp. Ther. 2011, 338, 559-567.

[58] J. Marschallinger, I. Schäffne, B. Klein, R. Gelfert, F. J. Rivera, S. Illes, L. Grassner, M. Janssen, P. Rotheneichner, C. Schmuckermair, R. Coras, M. Boccazzi, M. Chishty, F. B. Lagler, M. Renic, H. C. Bauer, N. Singewald, I. Blümcke, U. Bogdahn, S. Couillard-Despres, D. Chichung Lie, M. P. Abbracchio, L. Aigner, Nat. Commun. 2015, 6, 8466

[59] H. Ren, I. J. Orozco, Y. Su, S. Suyama, R. Gutiérrez-Juárez, T. L. Horvath, S. L. Wardlaw, L. Plum, O. Arancio, D. Accili, Cell 2012, 149, 1314-1326.

[60] C. Temporini, S. Ceruti, E. Calleri, S. Ferrario, R. Moaddel, M. P. Abbrac chio, G. Massolini, Anal. Biochem. 2009, 384, 123-129.

[61] E. Calleri, S. Ceruti, G. Cristalli, C. Martini, C. Temporini, C. Parravicini, R. Volpini, S. Daniele, G. Caccialanza, D. Lecca, C. Lambertucci, M. L. Trincavelli, G. Marucci, I. W. Wainer, G. Ranghino, P. Fantucci, M. P. Abbracchio, G. Massolini, J. Med. Chem. 2010, 53, 3489-3501.

[62] M. Köse, K. Ritter, K. Thiemke, M. Gillard, E. Kostenis, C. E. Müller, ACS Med. Chem. Lett. 2014, 5, 326-330.

[63] A. Maekawa, B. Balestrieri, K. F. Austen, Y. Kanaoka, Proc. Natl. Acad. Sci. USA 2009, 106, 11685-11690.

Received: September 1, 2016

Revised: October 17, 2016

Published online on II, 0000 


\section{MINIREVIEWS}

Potential CNS champion: Although the controversy regarding the ligands that interact with GPR17 still remains unclarified, it is well known that this receptor is involved in the differentiation of neuronal cells, the myelination process, and repair mechanisms following a brain insult. Clearly, GPR17 could represent a novel therapeutic target for the treatment of demyelinating disorders and development of promising anti-ischemic agents.

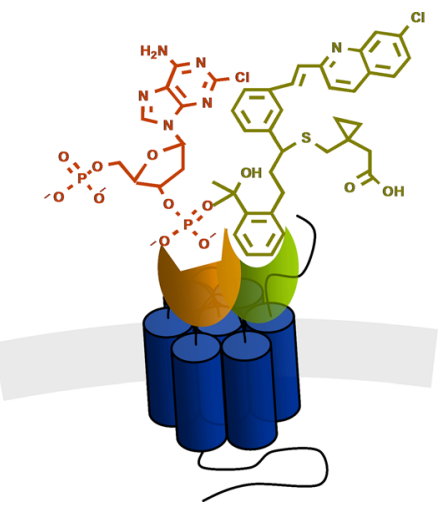

G. Marucci, D. Dal Ben, C. Lambertucci,

C. Santinelli, A. Spinaci, A. Thomas,

R. Volpini, M. Buccioni*

The G Protein-Coupled Receptor GPR17: Overview and Update 